\title{
Clinical, neuroelectrophysiological and muscular pathological analysis of chronic progressive external ophthalmoplegia
}

\author{
HAIDONG LV, QIANQIAN QU, HAIYAN LIU, QI QIAN, XIANZHAO ZHENG and YAN ZHANG \\ Department of Neurology, The People's Hospital of Jiaozuo City, Jiaozuo, Henan 454002, P.R. China
}

Received June 26, 2019; Accepted March 10, 2020

DOI: $10.3892 /$ etm.2020.8822

\begin{abstract}
The aim of the present study was to explore the clinical, neuroelectrophysiological and muscular pathological characteristics of chronic progressive external ophthalmoplegia (CPEO) and to improve the understanding of CPEO. Clinical manifestations, neuroelectrophysiology and pathological features of muscle biopsies from 12 patients with CPEO were retrospectively analyzed. The average age of onset for the 12 patients ( 6 males and 6 females) was 17.2 years. All patients had different degrees of blepharoptosis. A total of 11 patients experienced ocular dyskinesia, but diplopia was rare. Electrophysiological testing in 12 patients revealed abnormal changes in 6 patients, including 4 patients with a myogenic lesion, 1 patient with a neurogenic lesion, and 1 patient with mixed myogenic/neurogenic lesions. Two patients had slow sensory nerve conduction velocity. Muscle biopsies in 12 patients demonstrated ragged-red, irregular and broken fibers in 11 patients through Gomori trichrome and hematoxylin and eosin (H\&E) staining, increased lipid levels in some muscle fibers in 4 patients through Oil Ped O staining and abnormal distribution of type I and II muscle fibers in 3 patients through ATPase staining. Electron microscopy in 5 patients showed an increased number of mitochondria and abnormal mitochondrial aggregation between submucosa and myofibrils in 4 patients. These findings suggest that the possibility of CPEO should be considered if patients present with obvious extraocular muscle paralysis without diplopia. Furthermore, the identification of ragged-red fibers by Gomori trichrome and H\&E staining of muscle biopsies from patients is an important basis for the diagnosis of CPEO.
\end{abstract}

\section{Introduction}

Chronic progressive external ophthalmoplegia (CPEO) is a mitochondrial disease characterized by chronic progressive

Correspondence to: Dr Haidong Lv, Department of Neurology, The People's Hospital of Jiaozuo City, 267 Jiefang Middle Road, Jiaozuo, Henan 454002, P.R. China

E-mail: hnlhd666@163.com

Key words: chronic progressive external ophthalmoplegia, neuroelectrophysiology, muscle pathology, diagnosis blepharoptosis, ocular dyskinesia, ptosis, limited of eye movement and bulbar muscle weakness of varied severity $(1,2)$. The condition typically appears in adults aged 18-40 years and slowly worsens over time. Limb involvement is rarely observed with CPEO, and patients may only develop mild limb weakness. Therefore, the disease is easily misdiagnosed as ocular myasthenia gravis, oculopharyngeal muscular dystrophy, Fisher syndrome or other ophthalmic diseases (3). Mitochondria are cellular organelles found in all nucleated human cells. CPEO can be caused by mitochondrial DNA (mtDNA) point mutations, a single large scale mtDNA deletions, duplications or multiple mtDNA deletions, secondary to nuclear mutations to genes including ANT1, POLG1, POLG2, OPA1, C10orf2 and SLC25A4 (3,4). In total, 70\% of patients with CPEO were found to carry single large-scale mtDNA deletions, whilst a small number of patients have also been found to carry mtDNA point mutations (3-5). Skeletal muscle biopsies are used to confirm the diagnosis of CPEO, where $>2 \%$ of the ragged red fibres (RRF) in skeletal muscle biopsies are strongly suggestive of mitochondrial cytopathy (6).

CPEO is a rare disorder with a low prevalence. In the 20 years between January 1997 and January 2018, only 12 patients with CPEO were encountered among a total of 1,060 muscle biopsies that were performed at the People's Hospital of Jiaozuo City. In the present study, the clinical data of the 12 patients with CEPO were retrospectively analyzed, with the aim of investigating the clinical manifestations, neuroelectrophysiology and muscle pathology of patients with CEPO, while improving the current understanding of CPEO and promoting early diagnosis.

\section{Materials and methods}

Ethics statement. The present study was approved by the ethics committee of the People's Hospital of Jiaozuo City (Jiaozuo, China). All patients have provided written informed consent for publication.

Subjects. This retrospective study summarized and analyzed the clinical data of 12 patients who had been diagnosed with CPEO from a total of 1,060 muscle biopsies performed in the People's Hospital of Jiaozuo City between January 1997 and January 2018. There were 6 males and 6 females, aged between 9 and 56 years, with an average age of 32 years. The disease duration was 2-25 years, with an average of 13 years. The onset age was 7-35 years, with an average of 17.2 years. 
Electrophysiological examination. Concentric needle electromyography as well as sensory and motor nerve conduction velocities in all the patients were measured using an Evoked Potential/Electromyography Measuring System (MEB-9200K; Nihon Kohden Corporation). Deltoid, biceps brachii, iliopsoas, gluteus maximus, vastus medialis, tibialis anterior and gastrocnemius muscles were selected for electromyography. Data were recorded and analyzed, including: i) spontaneous potential during the quiet period (occurrence in more than two sites was considered an abnormality); ii) time limit, amplitude and percentage of polyphasic waves of 20 motor unit action potentials during contractions at small forces; and iii) waveform and peak-to-peak amplitude of the recruitment potential during contractions at large forces. Conduction velocities of the median nerve, ulnar nerve, common peroneal nerve, posterior tibial nerve and sural nerve were determined. The ulnar nerve and axillary nerve were selected for repetitive nerve stimulation.

Pathological examination. All the patients underwent open biopsy after local anesthesia, including biceps brachii in 8 cases, deltoid in 1 case and quadriceps femoris in 3 cases. A portion of each muscle specimen was frozen in liquid nitrogen, and sliced into $8-\mu \mathrm{m}$ frozen sections. Sections were subjected to conventional histological, enzyme and histochemical staining, including hematoxylin and eosin (H\&E), modified Gomori trichrome, reduced nicotinamide adenine dinucleotide tetrazolium reductase (NADH-TR), oil red O, periodic acid-Schiff reaction and ATPase ( $\mathrm{pH} 4.2,4.3,10.6$ and 10.8). Results were visualized using a light microscope (magnifications, $\mathrm{x} 200$ and $\mathrm{x} 400)$.

$H \& E$ staining. Frozen sections were first stained with hematoxylin solution for 5-10 min, rinsed under running water for $5 \mathrm{~min}$ and stained with eosin solution for $3 \mathrm{~min}$ at room temperature. The sections were then dehydrated with an alcohol gradient, cleared in xylene and mounted in neutral gum.

Modified Gomori trichrome staining. Frozen sections were immersed in hematoxylin solution for 5-10 $\mathrm{min}$ at room temperature, rinsed under running water for $5 \mathrm{~min}$, and then immersed in Gomori trichrome solution for $30 \mathrm{~min}$ at room temperature. After rinsing with distilled water, the sections were dehydrated with an alcohol gradient, cleared in xylene and mounted in neutral gum.

$N A D H-T R$ staining. Frozen sections were incubated with NADH-TR staining solution for $30 \mathrm{~min}$ in a thermostat at $37^{\circ} \mathrm{C}$, rinsed with distilled water, air-dried and finally sealed with glycerin gelatin.

Oil Red $O$ staining. Frozen sections were stained in Oil Red O staining solution for $30 \mathrm{~min}$ at room temperature. Following rinsing with running water for $5 \mathrm{~min}$, sections were counterstained with hematoxylin solution for $1 \mathrm{~min}$ at room temperature, rinsed with running water for $2 \mathrm{~min}$, and sealed with glycerin gelatin.

Periodic acid-Schiff reaction staining. Frozen sections were fixed in Carnoy's solution for $10 \mathrm{~min}$ at room temperature.

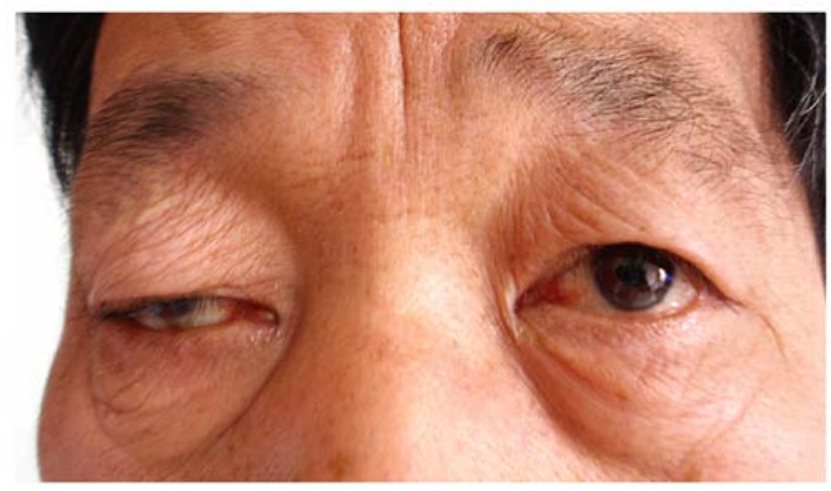

Figure 1. In a patient with blepharoptosis, the right eyelid covered half of the pupil, with binocular fixation in the central position.

Following rinsing with running water for $5 \mathrm{~min}$, the sections were immersed in $1 \%$ periodic acid solution for $5 \mathrm{~min}$, rinsed with distilled water for $2 \mathrm{~min}$, and then immersed in Schiff solution for $10 \mathrm{~min}$ at room temperature. After washing with running water for $5 \mathrm{~min}$, sections were counterstained with hematoxylin solution for 2-3 $\mathrm{min}$ at room temperature, and rinsed with running water for $5 \mathrm{~min}$. Then the sections were dehydrated with an alcohol gradient, cleared in xylene and mounted in neutral gum.

ATPase staining. Two $8-\mu \mathrm{m}$ frozen sections per patient were incubated with sodium acetate solution $(\mathrm{pH} 4.2$ and $\mathrm{pH} 4.3$ ) for $10 \mathrm{~min}$ at room temperature. Another two, $8-\mu \mathrm{m}$ frozen sections were incubated with calcium barbital solution (pH 10.6 and $\mathrm{pH} 10.8$ ) at $37^{\circ} \mathrm{C}$ for $10 \mathrm{~min}$. The four sections were then rinsed with running water for $5 \mathrm{~min}$ and incubated with $\mathrm{ATP} /$ calcium barbital solution $(\mathrm{pH} 9.4)$ at $37^{\circ} \mathrm{C}$ for $30 \mathrm{~min}$. They were then incubated in $2 \%$ cobalt chloride for $3 \mathrm{~min}$ at room temperature, rinsed with distilled water, immersed in $1 \%$ ammonium sulfide for $3 \mathrm{~min}$ at room temperature, and rinsed with running water for $5 \mathrm{~min}$.

Electron microscopic examination. Another portion of each muscle specimen was fixed in $2.5 \%$ glutaraldehyde for $24 \mathrm{~h}$ at room temperature; washed three times with sodium dimethylarsenate buffer; post-fixed in $1 \%$ osmic acid (in sodium dimethylarsenate buffer) for $2 \mathrm{~h}$ at room temperature; dehydrated with either an alcohol or acetone series; and then embedded in epoxy resin 618 at $37^{\circ} \mathrm{C}$ for $24 \mathrm{~h}$ and at $60^{\circ} \mathrm{C}$ for $48 \mathrm{~h}$. The specimens were sliced into ultrathin sections $(60 \mathrm{~nm})$ using a glass knife. The sections were stained with uranyl acetate at room temperature for $20 \mathrm{~min}$, rinsed thoroughly with water and stained with lead citrate at room temperature for $20 \mathrm{~min}$, following which these sections were dried and observed using a H-7500 transmission electron microscope (magnification, x10,000; Hitachi, Ltd.).

\section{Results}

Clinical characteristics. All 12 patients had varying degrees of chronic progressive blepharoptosis. The eyelids covered half of the pupil in 6 patients, two-thirds of the pupil in 4 patients and the whole pupil in 2 patients (Fig. 1). Of the 12 patients, 11 patients suffered from obvious ocular dyskinesia (including 
8 patients with binocular fixation in the central position) and 3 patients had obvious binocular horizontal and vertical eye movement disorders. All patients had a normal pupil size and a light reflex without diplopia. The disease duration was between 2-25 years. The onset age was 7-35 years, with an average of 17.2 years. Four patients developed mild limb weakness several years after onset, and 1 patient displayed swallowing difficulties.

Neuroelectrophysiological changes. Needle electromyography in 12 patients revealed abnormal changes in 6 patients, including myogenic lesions in 4 patients, a neurogenic lesion in 1 patient and mixed myogenic/neurogenic lesions in 1 patient. A decreased sensory nerve conduction velocity was observed in 2 patients. One of these 2 patients had a decreased sensory conduction velocity of the median nerve and posterior tibial nerve, and the other had a decreased sensory conduction velocity of the sural nerve. Negative symptoms were determined in 12 patients after repetitive stimulation.

Pathological changes in muscles. H\&E staining demonstrated that among the 12 patients, muscle fiber size was normal in 10 patients and a few angular atrophic muscle fibers were visible in 2 patients. In 11 patients, basophilic muscle fibers were irregularly scattered in the sarcoplasm and some fibers were broken (Fig. 2A). No degeneration or necrosis of muscle fibers and no infiltration of inflammatory cells were evident in any of the 12 patients. Modified Gomori trichrome staining revealed several typical ragged-red fibers (Fig. 2B). NADH-TR staining showed that the edges of the muscle fibers were deeply stained in 11 of 12 patients (Fig. 2C). The Oil Red O staining demonstrated that among 12 patients, there was a marked increase in lipids in some of the muscle fibers from 4 patients, predominantly in type I muscle fibers (Fig. 2D). ATPase staining demonstrated that type I and II muscle fibers were distributed normally in 9 patients. Both types of muscle fiber were abnormally distributed in 3 patients, which showed fiber-type grouping, where the type I fibers were more prevalent (Fig. 2E). Periodic acid-Schiff reaction staining did not reveal any abnormalities in the 12 patients (Fig. 2F).

Transmission electron microscopy showed that among the 5 patients tested, there were increased mitochondria and abnormal mitochondrial aggregation with varied shapes and sizes observed between the subsarcolemma and myofibrils in 4 of the 5 patients (Fig. 3).

\section{Discussion}

CPEO is a rare mitochondrial myopathy that can occur at any age, but is commonly found in children and young people (7). Most of the cases are sporadic and familial cases are rare (8). In the present study, the 12 patients were sporadic cases, with an onset age of 7-35 years and an average age of onset of 17.2 years. The average onset age was older compared with that of previous studies $(9,10)$. Patients with CPEO were mainly characterized by progressive blepharoptosis and ocular dyskinesia (7). In the present study, the 12 patients who were analyzed suffered from unilateral or bilateral blepharoptosis, then gradually developed ocular dyskinesia followed by binocular fixation in the central position. Diplopia rarely occurs during the clinical progression of CPEO (11). In the present study, only one case presented short-term and mild diplopia. Patients may develop severe extraocular muscle paralysis without diplopia since the bilateral extraocular muscles are involved and paralysis of the extraocular muscles progresses slowly as extraocular muscles can compensate for each other (11). The main clinical symptoms of CPEO are blepharoptosis and paralysis of the extraocular muscles in the early stages. Limb movement disorders rarely occur, so patients are often first seen by an ophthalmologist $(7,12)$. Among the 12 patients included in the present study, 9 were first treated by an ophthalmologist and 5 patients were diagnosed with weakness of the levator palpebrae superioris muscle. Three patients were treated with two eyelid lifting surgeries. CPEO is easily misdiagnosed as ocular myasthenia gravis in the clinic (12). In the present study, 8 patients were initially diagnosed with ocular myasthenia gravis and 6 patients received short-term treatment with pyridostigmine bromide, but their clinical symptoms did not improve. The causes of clinical misdiagnosis are potentially due to a lack of awareness of CPEO by clinicians and the failure to consider CPEO as the diagnosis. Clinicians may only pay attention to ptosis in the first diagnosis, and not notice or consider the signs of binocular eye movement disorders. In myasthenia gravis, blepharoptosis is more serious than ocular dyskinesia, and diplopia is obvious. Moreover, the symptoms of diplopia tend to fluctuate, with the presence of fatigue. However, paralysis of the extraocular muscles in patients with CPEO is often more prominent than blepharoptosis, and eventually bilateral eyeballs are fixed in the central position, while diplopia symptoms are not clear. Taken together, a diagnosis of CPEO should be considered first in patients with extraocular muscle paralysis which presents as more serious than blepharoptosis, if the neostigmine test shows a negative result.

Neuroelectrophysiological testing is a conventional examination for the diagnosis of neuromuscular diseases. Repetitive nerve stimulation is an effective method for differentiating CPEO from other neuromuscular diseases $(12,13)$. Repetitive nerve stimulation demonstrated negative results in all 12 patients in the present study, which did not support neuromuscular junction lesions. Needle electromyography revealed abnormal changes in 6 patients, including myogenic lesion in 4 patients, a neurogenic lesion in 1 patient and mixed myogenic/neurogenic lesions in 1 patient. These results indicated that although no obvious limb weakness symptoms were observed in the clinic, some abnormal changes were evident from the electrophysiological examination. Furthermore, besides myogenic lesions, a neurogenic lesion or mixed lesions were also be seen in individual patients. In nerve conduction measurements, 2 patients demonstrated decreased sensory nerve conduction velocity. This suggests that patients with CPEO may also have peripheral nerve damage, which has rarely been reported in previous studies $(11,12)$.

Muscle biopsies are an important method for the diagnosis of mitochondrial myopathy in CPEO. In 1963, Engel and Cunningham (14) first discovered ragged-red fibers using modified Gomori trichrome staining. In 1977, Shapira et al (15) proposed mitochondrial encephalomyopathies. In 1988, Yamamoto and Nonaka (16) described CPEO, which provided an in-depth understanding of the muscle pathology of 


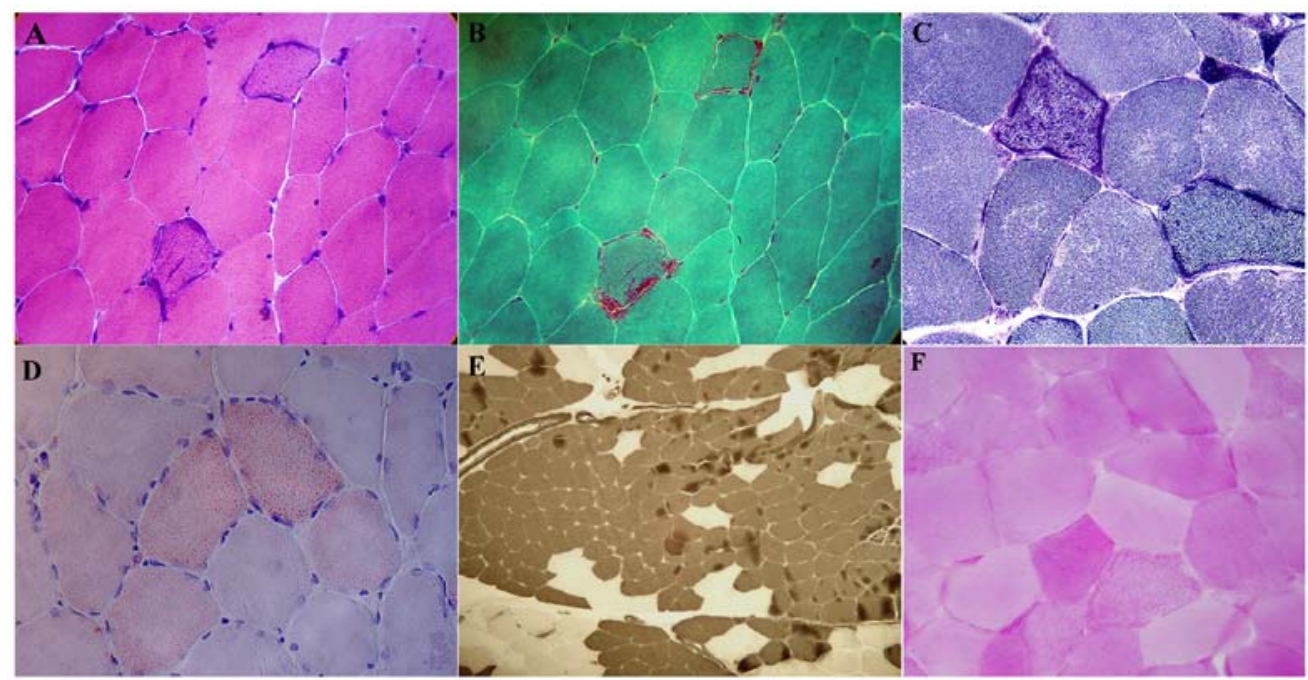

Figure 2. Representative staining images of tissues from patients with chronic progressive external ophthalmoplegia. (A) Hematoxylin and eosin staining of muscle biopsies showed normally sized muscle fibers, abnormal scattered deeply-stained muscle fibers with basophilic sarcoplasm and some broken muscle fibers (magnification, x400). (B) Modified Gomori trichrome staining of muscle biopsies showed a number of typical ragged-red fibers in the muscle tissue (magnification, $\mathrm{x} 400$ ). (C) Nicotinamide adenine dinucleotide tetrazolium reductase staining of muscle biopsies showed that the edges of the muscle fibers were deeply stained blue (magnification, x400). (D) Oil Red O staining of muscle biopsies showed a significant increase in red particles in some of the muscle fibers, suggesting an increase in lipids (magnification, $\mathrm{x} 400$ ). (E) ATPase staining of muscle biopsies showed abnormal distribution of type I and II muscle fibers which appear as fiber-type grouping (magnification, x200). (F) Periodic acid-Schiff reaction staining of muscle biopsies revealed no abnormalities (magnification, $\mathrm{x} 400$ ).

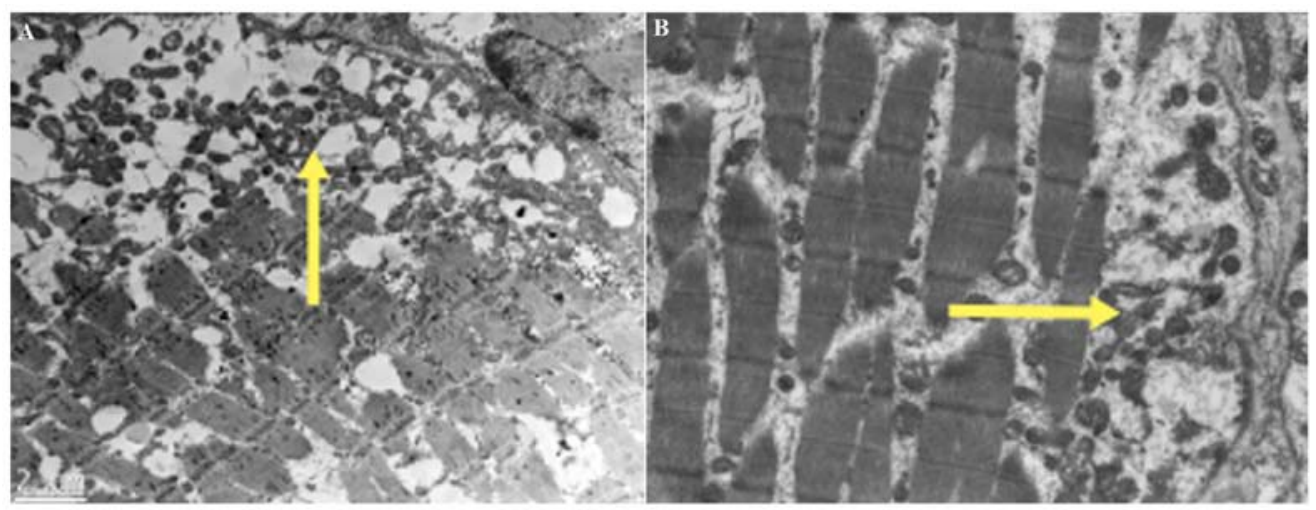

Figure 3. Representative transmission electron microscopy images of patients with chronic progressive external ophthalmoplegia. (A) Massive abnormal subsarcolemma mitochondrial aggregation in a patient, as shown by the yellow arrow. Lead-uranium double staining (magnification, x12,500). (B) Massive abnormal mitochondrial aggregations were observed, with varied shapes and sizes between subsarcolemmas and myofibrils, as demonstrated by the yellow arrow. Lead-uranium double staining (magnification, $x 12,500$ )

mitochondrial diseases. When ragged-red fibers are found in muscle fibers, it indicates that there are a large number of abnormally proliferating and aggregated mitochondria in the muscle fibers (6). It is generally considered that $>2 \%$ of the total fibers presenting as ragged-red fibers is an important basis for the diagnosis of mitochondrial myopathy (6). In the present muscle biopsies, the proportion of ragged-red fibers was $>2 \%$. Thus, CPEO could be diagnosed by this in combination with the clinical manifestations. In addition to Gomori trichrome staining, $\mathrm{H} \& \mathrm{E}$, Oil Red $\mathrm{O}$ and ATPase staining were also performed. Scattered, abnormal muscle fibers with deeply stained coarse granules were visible in the H\&E-stained sections. The 11 patients who had ragged-red fibers on Gomori trichrome staining also had abnormal deeply basophilic staining of muscle fibers as observed by H\&E staining. Oil Red O staining was positive in 4 patients, suggesting that CPEO could be combined with abnormal lipid metabolism, resulting in lipid deposition in muscle fibers. ATPase staining demonstrated the abnormal distribution of type I and II muscle fibers in 3 patients, where the fibers grouped together with predominantly type 1 fibers. This abnormal distribution of muscle fibers suggests that there may be peripheral nerve damage in patients with CPEO. In one of the patients with abnormally distributed muscle fibers, electromyography tests showed neurogenic damage to some of the muscles examined and there was a slightly decreased conduction velocity of the peripheral nerves. These data also support that CPEO could be accompanied by peripheral nerve damage.

With the rapid development of molecular tests, many mtDNA fragment losses and point mutations have been identified in patients with CPEO (17-19). Sundaram et al (20) performed muscle biopsies and gene detection in 45 patients with CPEO, and found neurogenic atrophy in seven biopsies. Mitochondrial gene mutations were found in 10 of the 
11 patients who presented with a normal muscle biopsy. Lehmann et al (21) found that peripheral nerve involvement was associated with multiple deletions in mtDNA fragments. Gene detection has become a new diagnostic basis for CPEO, and should be conducted in future studies to identify the loss of mtDNA fragments and mutation sites.

It should be noted that the present study has limitations. Firstly, the sample size is small. Since CPEO is a rare disorder, inclusion of more patients with CPEO is very difficult, but the inclusion of more patients in future multi-center studies would be useful to clarify the clinical feature of different types of CPEO. Secondly, a number of possible factors were not analyzed, for example the disease duration and age of the patients could have affected the results and needs to be further investigated. Finally, given the importance of gene detection in the diagnosis of CPEO, further studies are needed to investigate the genetic abnormalities associated with patients with CPEO, to further improve the molecular-pathological diagnosis of CPEO.

In conclusion, the present study suggests that if patients present with obvious extraocular muscle paralysis and do not have symptoms of diplopia, the possibility of CPEO should be considered. Ragged-red fibers identified by Gomori trichrome staining and the $\mathrm{H} \& \mathrm{E}$ staining of muscle biopsies from patients is essential for confirming the diagnosis of CPEO.

\section{Acknowledgements}

Not applicable.

\section{Funding}

No funding was received.

\section{Availability of data and materials}

The datasets used and/or analyzed during the current study are available from the corresponding author on reasonable request.

\section{Authors' contributions}

HL contributed to the study conception and design. QQQ, HL, QQ, XZ, YZ contributed to the acquisition, analysis and interpretation of data. All authors read and approved the final manuscript.

\section{Ethics approval and consent to participate}

The present study was approved by the Ethics Committee of the People's Hospital of Jiaozuo City. All patients have given written informed consent.

\section{Patient consent for publication}

All patients have given written informed consent for publication.

\section{Competing interests}

The authors declare that they have no competing interests.

\section{References}

1. López-Gallardo E, López-Pérez MJ, Montoya J and Ruiz-Pesini E: CPEO and KSS differ in the percentage and location of the mtDNA deletion. Mitochondrion 9: 314-317, 2009.

2. Negro R, Zoccolella S, Dell'aglio R, Amati A, Artuso L, Bisceglia L, Lavolpe V, Papa S, Serlenga L and Petruzzella V: Molecular analysis in a family presenting with a mild form of late-onset autosomal dominant chronic progressive external ophthalmoplegia. Neuromuscul Disord 19: 423-426, 2009.

3. Murdock J, Thyparampil PJ and Yen MT: Late-onset development of eyelid ptosis in chronic progressive external ophthalmoplegia: A 30-year follow-up. Neuroophthalmology 40: 44-46, 2016.

4. Filosto M, Mancuso M, Nishigaki Y, Pancrudo J, Harati Y, Gooch C, Mankodi A, Bayne L, Bonilla E, Shanske S, et al: Clinical and genetic heterogeneity in progressive external ophthalmoplegia due to mutations in polymerase $\gamma$. Arch Neurol 60: 1279-1284, 2003.

5. Brandon BR, Diederich NJ, Soni M, Witte K, Weinhold M, Krause $M$ and Jackson S: Autosomal dominant mutations in POLG and C10orf2: Association with late onset chronic progressive external ophthalmoplegia and Parkinsonism in two patients. J Neurol 260: 1931-1933, 2013.

6. Walker UA, Collins S and Byrne E: Respiratory chain encephalomyopathies: A diagnostic classification. Eur Neurol 36: 260-267, 1996.

7. Chaturvedi S, Bala K, Thakur R and Suri V: Mitochondrial encephalomyopathies: Advances in understanding. Med Sci Monit 11: RA238-RA246, 2005.

8. Biousse V and Newman NJ: Neuro-ophthalmology of mitochondrial diseases. Curr Opin Neurol 16: 35-43, 2003.

9. Bing Q, Hu J, Li N, Zhao Z, Shen HR and Yuan HH: Clinical and pathological features of chronic progressive external ophthalmoplegia. Linchuang Shenjingbingxue Zazhi 22: 175-177, 2009 (In Chinese).

10. Sun L, Lu JH and Lu ZZ: Clinical manifestations, pathological changes and diagnosis of chronic progressive external ophthalmoplegia (twelve cases attached). Fudan Daxue Yixueban 36: 212-215, 2009 (In Chinese).

11. Wallace DK, Sprunger DT, Helveston EM and Ellis FD: Surgical management of strabismus associated with chronic progressive external ophthalmoplegia. Ophthalmology 104: 695-700, 1997.

12. Wu JL, Yan CZ, Wang QZ, Liu SP, Gao SQ, Zhang YQ and Li DN: Chronic progressive external ophthalmophegia: Clinical and pathological analysis of 22 cases. Zhonghua Shenjinke Zazhi 38: 737-740, 2005 (In Chinese).

13. Fournier E and Tabti N: Clinical electrophysiology of muscle diseases and episodic muscle disorders. Handb Clin Neurol 161: 269-280, 2019.

14. Engel WK and Cunningham GG: Rapid examination of muscle tissue. An improved trichrome method for fresh-frozen biopsy sections. Neurology 13: 919-923, 1963.

15. Shapira Y, Harel S and Russell A: Mitochondrial encephalomyopathies: A group of neuromuscular disorders with defects in oxidative metabolism. Isr J Med Sci 13: 161-164, 1977.

16. Yamamoto $M$ and Nonaka I: Skeletal muscle pathology in chronic progressive external ophthalmoplegia with ragged-red fibers. Acta Neuropathol 76: 558-563, 1988.

17. Lee SJ, Na JH, Han J and Lee YM: Ophthalmoplegia in mitochondrial disease. Yonsei Med J 59: 1190-1196, 2018.

18. Sachdev A, Fratter C and McMullan TF: Novel mutation in the RNASEH1 gene in a chronic progressive external ophthalmoplegia patient. Can J Ophthalmol 53: e203-e205, 2018.

19. Viscomi C and Zeviani M: MtDNA-maintenance defects: Syndromes and genes. J Inherit Metab Dis 40: 587-599, 2017.

20. Sundaram C, Meena AK, Uppin MS, Govindaraj P, Vanniarajan A, Thangaraj K, Kaul S, Kekunnaya R and Murthy JM: Contribution of muscle biopsy and genetics to the diagnosis of chronic progressive external opthalmoplegia of mitochondrial origin. J Clin Neurosci 18: 535-538, 2011.

21. Lehmann D, Kornhuber ME, Clajus C, Alston CL, Wienke A, Deschauer M, Taylor RW and Zierz S: Peripheral neuropathy in patients with CPEO associated with single and multiple mtDNA deletions. Neurol Genet 2: e113, 2016. 